\title{
Powering up a failing heart
}

Reporting in Science, Malik et al. show that a small molecule that directly activates the contractility of cardiac myosin by increasing its ATPase activity improves cardiac function in systolic heart failure. This approach exploits a different mechanism of action compared to current therapies - such as beta blockers and phosphodiesterase inhibitors - which indirectly increase cardiac contractility by increasing the levels of calcium via activation of second messenger signalling pathways, but are also associated with negative effects such as arrhythmias and hypotension.

The small molecule - omecamtiv mecarbil - was optimized from a hit that was identified by a high-throughput screen for selective, direct activators of the cardiac sarcomere. Omecamtiv mecarbil has already demonstrated efficacy in dogs with systolic heart failure, and so this paper describes the mechanistic principles behind its activity.

Omecamtiv mecarbil selectively activates the S1 domain of cardiac myosin but not of other muscle myosins (such as smooth muscle and skeletal muscle isoforms), and its proposed site of action is a region on the $\mathrm{S} 1$ domain that is a convergence point between the nucleotidebinding pocket and the lever arm that is responsible for the power stroke. This site therefore allows omecamtiv mecarbil to allosterically modulate both the enzymatic and mechanical properties of the cardiac myosin motor. In detail, the compound accelerates the transition rate between the weakly actin-bound state to a strongly actin-bound state, as indicated by the acceleration of actin-dependent phosphate release. This step is rate-limiting in the actin-myosin ATPase cycle in contracting muscle, and thus overall ATP turnover is increased; it occurs in concert with the force-producing power stroke. Consequently, the number of myosins that bind to actin in a force-producing manner is increased, thereby increasing force output and improving contractile function. Importantly, as previously reported, omecamtiv mecarbil is able to increase cardiac contractility without affecting calcium homeostasis or overall myocardial oxygen consumption.

These data demonstrate the feasibility of selectively targeting a subset of myosins with a small molecule to increase the contractile function of the heart, thereby avoiding the side effects that are associated with current therapeutics. Omecamtiv mecarbil has completed Phase I and Phase IIa trials, and a Phase IIb trial is anticipated to begin in the first half of this year.

\section{Man Tsuey Tse}

ORIGINAL RESEARCH PAPER Malik, F. I. et al. Cardiac myosin activation: a potential therapeutic approach for systolic heart failure. Science 331, 1439-1443 (2011)

FURTHER READING Kaye, D. M. \& Krum, H.

Drug discovery for heart failure: a new era or the end of the pipeline? Nature Rev. Drug Discov. 6 , 127-139 (2007). 